\title{
HSP70s Enhance a Phytophthora infestans Effector-Induced Cell Death via an MAPK Cascade in Nicotiana benthamiana
}

\author{
Joo Hyun Lee ${ }^{1}$, So Eui Lee', Soohyun Oh'1, Eunyoung Seo', and Doil Choi ${ }^{1,2,+}$ \\ ${ }^{1}$ Department of Plant Science and Plant Genomics and Breeding Institute, Institute of Agricultural Biotechnology, College of \\ Agriculture and Life Science, Seoul National University, Seoul, 08826, Republic of Korea; and ${ }^{2}$ Institute of Seed Biotechnology, \\ Institute of Green Bio Science and Technology, Seoul National University, Pyeongchang, 25354, Republic of Korea
}

Accepted 13 November 2017.

\begin{abstract}
A destructive pathogen, Phytophthora infestans, secretes hundreds of effectors for successful survival in its host plants. The effectors modulate the plant defense system at diverse cellular compartments to take an advantage of pathogen survivals. A few research studies have shown the mode of action of each effector and their interacting proteins in plant cells. Here, we investigated the mode of action of a $P$. infestans effector, Pi23226, which induces cell death in Nicotiana benthamiana. To identify its host factors, we performed coimmunoprecipitation and liquid chromatography-mass spectrometry, and selected members of heat shock protein 70 (HSP70s) as candidates. These HSP70s, known to function as chaperones, were associated with Pi23226 in planta and accelerated Pi23226-induced cell death. Additionally, they were found to be involved in plant basal defense by suppressing the growth of $P$. infestans. We also found that specific components of a mitogen-activated protein kinase cascade were involved in Pi23226-induced cell death. Our findings show that HSP70s functions in defense systems by regulating effector-triggered cell death and by suppressing the growth of the pathogen. This suggests that host plants manipulate the ubiquitous proteins to detect pathogen effectors for functioning in the defense system.
\end{abstract}

Plants are frequently exposed to various pathogen invasions and have developed multilayered defense systems against diverse pathogens, including bacteria, viruses, fungi, and oomycetes. When pathogens invade plants by overcoming preformed defense systems such as waxy cuticles and phytoanticipins, plants recognize pathogen-associated molecular patterns (PAMP) and activate induced defense signaling (referred to as PAMP-triggered immunity), which accumulates reactive oxygen species (ROS), activates mitogen-activated protein kinase (MAPK) pathways, increases influx of calcium ions, and induces defense-related genes. Against plant-induced defenses, pathogens secrete specialized

Funding: This work was supported by a grant from the Agricultural Genome Center of the Next Generation Biogreen 21 Program of RDA (project number PJ01127501) and by a grant from the Ministry of Science, ICT, and Future Planning of the Korean government through the National Research Foundation (NRF-2015R1A2A1A01002327) to D. Choi.

${ }^{\dagger}$ Corresponding author: Doil Choi; E-mail: doil@snu.ac.kr

*The $\boldsymbol{e}$-Xtra logo stands for "electronic extra" and indicates that one supplementary table and five supplementary figures are published online.

@ 2018 The American Phytopathological Society molecules called effectors into plant cells to modulate the plant defense system. These effectors interact with several plant proteins, named host factors, at diverse cellular compartments to suppress the plant defense system. In response to activities of effectors, plants activate more prolonged and intense defense signaling (referred to as effector-triggered immunity) using resistance $(R)$ genes encoding intracellular nucleotide-binding and leucine-rich repeat receptors. As the result of interaction between effectors and the corresponding $\mathrm{R}$ proteins, localized cell death at the infection site, called hypersensitive response (HR), often occurs to disrupt pathogen spread (Jones and Dangl 2006). Although plants have evolved the corresponding $R$ genes against pathogen infections, the plant defense system is frequently challenged by new virulence traits which evolutionarily develop a diverse repertoire of effectors using rapid turnover and extensive expansion in their genomes (Cooke et al. 2012; Haas et al. 2009). Therefore, understanding the biochemical functions of effectors will provide insights to answer how pathogens manipulate host defense systems to establish disease.

A member of the oomycetes, Phytophthora infestans is the causal agent of late blight, which triggered the great Irish potato famine of the 1840s. To date, P. infestans is the most destructive pathogen threatening the economically important crop agriculture. Host species of the pathogen such as potato and tomato belong to the Solanaceae family. Worldwide yield losses of potato production by $P$. infestans is annually estimated to $\$ 6.7$ billion (Haverkort et al. 2008). However, the control of this pathogen is challenged because of its remarkable ability of rapid adaptation. In 2009, the first $P$. infestans genome has been published and provided massive information on its genome architecture and effectors (Haas et al. 2009). One type of cytosolic effectors, members of the RXLR family, are modular proteins containing an Arg-X-Leu-Arg (in which X represents any amino acid) motif required for delivery of the effectors into plant cells with an $\mathrm{N}$-terminal signal peptide for secretion and a C-terminal effector domain for biochemical activity.

In recent decades, several studies have presented RXLR effectors that modulate plant immunity by associating diverse plant proteins. One of the RXLR effector of $P$. infestans, Avr2, interacts with potato BSU-LIKE PROTEIN1 (BSL1) and mediates the interaction between BSL1 and an R protein (R2) in planta (Saunders et al. 2012). Another RXLR effector, Avrblb2, is known to interact with the plant membrane-associated ROMORIN1.3 and a protease, C14, and enhances the activity of pathogen growth (Bozkurt et al. 2011, 2014). HopI1, a virulence effector of Pseudomonas syringae, is known to associate with heat shock protein 70 (HSP70s), which functions as a 
chaperone in response to biotic and abiotic stimuli. HSP70s is essential for the virulent-enhancing activity of HopI1. When gene expression of HSP70s is suppressed, plants increase susceptibility against the pathogen (Jelenska et al. 2010). In pepper, cytoplasmic HSP70s physically interacts with the Xanthomonas effector, AvrBsT. As shown in virus-induced gene silencing (VIGS) analysis, HSP70s functions as a positive regulator of plant defense by affecting ROS accumulation, cell death response, levels of salicylic acid and jasmonic acid, as well as expression of defense-related genes (Kim and Hwang 2015). In addition, the Phytophthora infestans elicitor, INF1-induced HR cell death, disappears in HSP70s-silenced plants (Kanzaki et al. 2003). However, the molecular mechanisms of HSP70s in plant defense are still elusive. Several studies suggest that HSP70s, together with HSP90, might function upstream of the MAPK cascade or independently (Kanzaki et al. 2003).

In this study, we selected one $P$. infestans RXLR effector, Pi23226, for studying the mode of action in Nicotiana benthamiana. Pi23226 induces cell death and accelerates the growth of $P$. infestans. In the screening of potential host factors of Pi23226 using coimmunoprecipitation and liquid chromatography-mass spectrometry (LC-MS/MS), members of HSP70s were identified as associating with Pi23226 in planta. Furthermore, HSP70s members accelerate Pi23226-induced cell death and enhance plant defense against $P$. infestans.

\section{RESULTS}

\section{A $P$. infestans effector, $\mathbf{P i 2 3 2 2 6}$, induces cell death in $N$. benthamiana.}

To investigate the role of Pi23226 expression in N. benthamiana, an agroinfiltration assay was performed. Because several previous studies revealed that effector domains at the $\mathrm{C}$ terminus are sufficient for activities (Bozkurt et al. 2011; Saunders et al. 2012), the effector domain of Pi23226 was cloned and introduced into a pGR106-based vector, pICH31160, for the analysis (hereafter Pi23226 pKw) (Nausch et al. 2012). Bax, a proapoptotic Bcl-2family protein, and mCherry, in the same vector with Pi23226, were used as a positive and negative control, respectively (Fig. 1A). A necrotic lesion at the infiltrated region of Pi23226 pKw was shown 3 days postinfection (dpi), similar to Bax-induced cell death, indicating that Pi23226 indeed induces cell death in N. benthamiana (Fig. 1B). To identify Pi23226's biochemical mechanism of action, three epitope FLAG tags were attached in front of the effector domain of Pi23226 and the construct was introduced into the $\mathrm{pKw}$ vector (3xFLAG:Pi23226). Agroinfiltration of the tagged Pi23226 also induced cell death at 4 dpi in $N$. benthamiana. The cell death was distinct at the infiltrated region of 3xFLAG:Pi23226 but became rather weaker compared with that of Pi23226 pKw (Fig. 1B).

\section{Pi23226 enhances pathogen growth}

\section{of $P$. infestans in $N$. benthamiana.}

Previous studies reported that diverse RXLR effectors of $P$. infestans, including Pi23226, were highly induced at 2 and 3 dpi after the pathogen was challenged and modulated the plant defense system to take advantage of pathogen colonization and reproduction (Cooke et al. 2012; King et al. 2014; McLellan et al. 2013; Zheng et al. 2014). Because effectors are likely expected to function positively for pathogen invasion or growth, we investigated whether Pi23226 acts as a virulence factor. However, because Pi23226 pKw induces cell death in $N$. benthamiana, it had a limitation for investigating whether Pi23226 can perform virulence-enhancing activity as an effector. In order to overcome this problem, we introduced Pi23226 into another vector, pCAMBIA2300-LIC (Kim et al. 2015). Pi23226 was expressed at the relevant level but was not sufficient to trigger cell death (Fig. 2A and B). When zoospores of $P$. infestans were inoculated on $N$. benthamiana leaves that were transiently expressed of Pi23226 on a half area, with an empty vector on the other half as a negative control, significantly larger diseased lesions were observed in areas expressing Pi23226 compared with the empty vector, indicating that Pi23226 functions positively in pathogen growth (Fig. 2C and D). Together with cell death results, Pi23226 having virulence-enhancing activity helps pathogen infection, while plants recognize the activity of $\mathrm{Pi} 23226$ and induce cell death to suppress the spread of pathogen growth.

\section{Identification of host target proteins interacting with Pi23226.}

To elucidate how Pi23226 triggers cell death in plant cells, possible host factors interacting with Pi23226 were screened. For this, $\alpha$-FLAG immunoprecipitation (IP) was performed

A
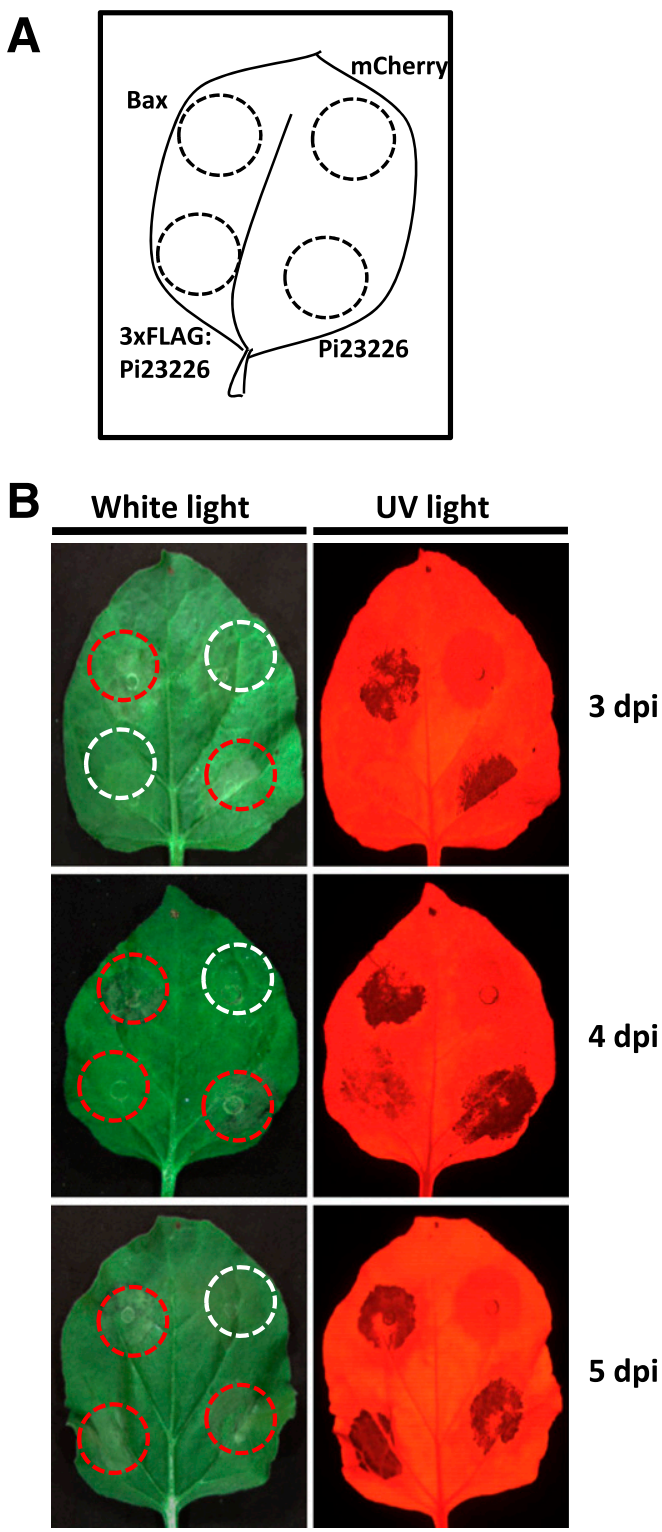

Fig. 1. Pi23226 induces cell death in Nicotiana benthamiana. A, Schematic drawing shows agroinfiltration of constructs for cell death phenotype analysis. Bax and mCherry were used as a positive control and a negative control for cell death response, respectively. B, Representative images show cell death induced by Pi23226. optical density at $600 \mathrm{~nm}$ of each construct was 0.4 . Images were taken 3,4 , and 5 days after agroinfiltration (dpi) under UV and white light. All experiments were performed in three biological replicates and representative results are shown. 
with 3xFLAG:Pi23226 agroinfiltrated in N. benthamiana at 2 dpi. After confirmed the appropriate amount of 3xFLAG:Pi23226 and its precipitates by $\alpha$-FLAG immunoblotting, host factor candidates were identified using LC-MS/MS (Supplementary Fig. S1). To avoid false positive selection, peptide sequences of which were detected in $\alpha$-FLAG IP with 3xFLAG:GFP-infiltrated were excluded. As a result, 20 peptide reads were identified by LC$\mathrm{MS} / \mathrm{MS}$, and 7 peptide sequences corresponded to distinct isoforms of heat shock protein 70 (HSP70s).

There were 64 genes containing HSP70s specific domain (PF00012) based on the $N$. benthamiana draft genome sequence. Among them, 47 genes containing more than 250 amino acids
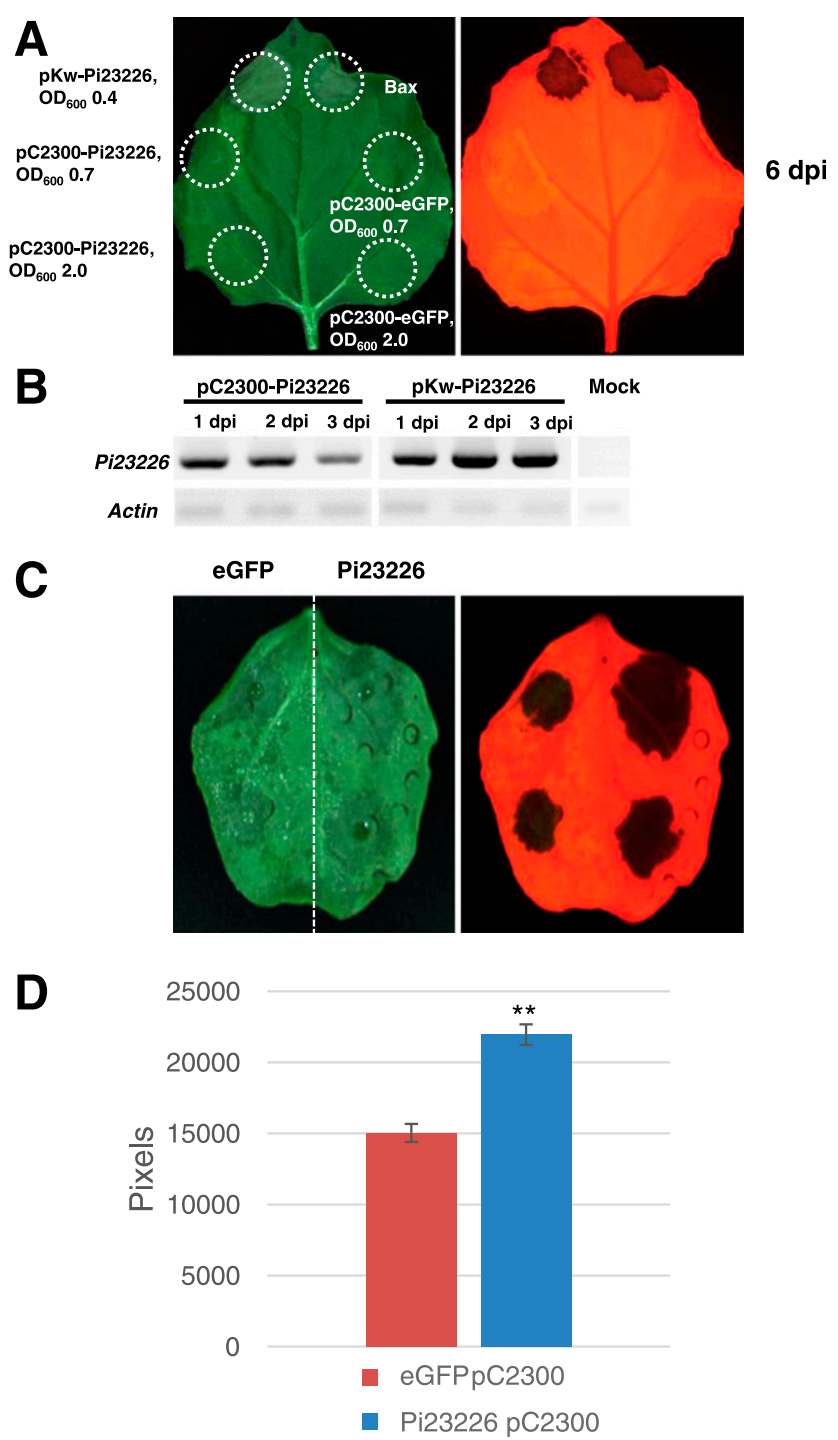

Fig. 2. Pi23226 enhances disease symptom when Phytophthora infestans infected in Nicotiana benthamiana. A, Cell death phenotypes of agroinfiltrated Pi23226 in different vectors. Pi23226 was introduced into pKw (a virus vector) and pC2300 (a binary vector). pC2300 Pi23226, which showed no cell death phenotype, was tested with optical density at $600 \mathrm{~nm}$ $\left(\mathrm{OD}_{600}\right)$ of 0.7 and 2.0 ; dpi = days postinfection. B, Gene expression of Pi23226 in different vectors. C, Images show disease symptom of $P$. infestans in $N$. benthamiana. Pi23226 pC2300 was transiently expressed on one-half of a leaf and enhanced green fluorescent protein (eGFP) pC2300 as a negative control on the other. Zoospores of $P$. infestans were placed and allowed to grow for approximately 4 to 5 days. D, Graph represents average lesion size of $P$. infestans at the transiently expressed sites. Sizes were measured by ImageJ and the relevance of difference was evaluated by $P$ value. All experiments were performed in more than three biological replicates and representative results are shown. were used for phylogenetic analysis. As a result, HSP70s members precipitated by 3xFLAG:Pi23226 were located broadly on the highly conserved area (Supplementary Fig. S2). In this study, two distinct isoforms of HSP70s (Niben101Scf12868g00008.1 and Niben101Scf00117g02019.1, hereafter named as NbHSP70s-1 and NbHSP70s-2, respectively) were selected for further study of the roles of NbHSP70s members in Pi23226-induced cell death response.

To confirm the interaction between 3xFLAG:Pi23226 and NbHSP70s members that occurs in planta, $\alpha$-FLAG co-IP experiments were conducted using 3xFLAG:Pi23226 and HSP70s tagged with green fluorescent protein (GFP) at the $\mathrm{N}$ terminus (referred to as GFP:NbHSP70s-1 and GFP:NbHSP70s-2, respectively). GFP:NbHSP70s-1 and GFP:NbHSP70s-2 were precipitated in the presence of FLAG:Pi23226 but not in its absence, whereas all constructs were detected in the appropriate input (Fig. 3A and B). These results indicate that GFP:NbHSP70s-1 and GFP:NbHSP70s-2 indeed interact with 3xFLAG:Pi23226 in planta.

HSP70s is essential for Pi23226-triggered cell death and enhances plant defense against $P$. infestans.

To understand the effect of HSP70s on Pi23226-induced cell death, coagroinfiltration of Pi23226 pKw and HSP7Os was performed in $N$. benthamiana. Although NbHSP70s-1 and NbHSP70s-2 themselves did not induce cell death, both NbHSP70s1 and NbHSP70s-2 accelerated Pi23226-induced cell death (Fig. 4A). To investigate whether the enhancement of Pi23226-induced

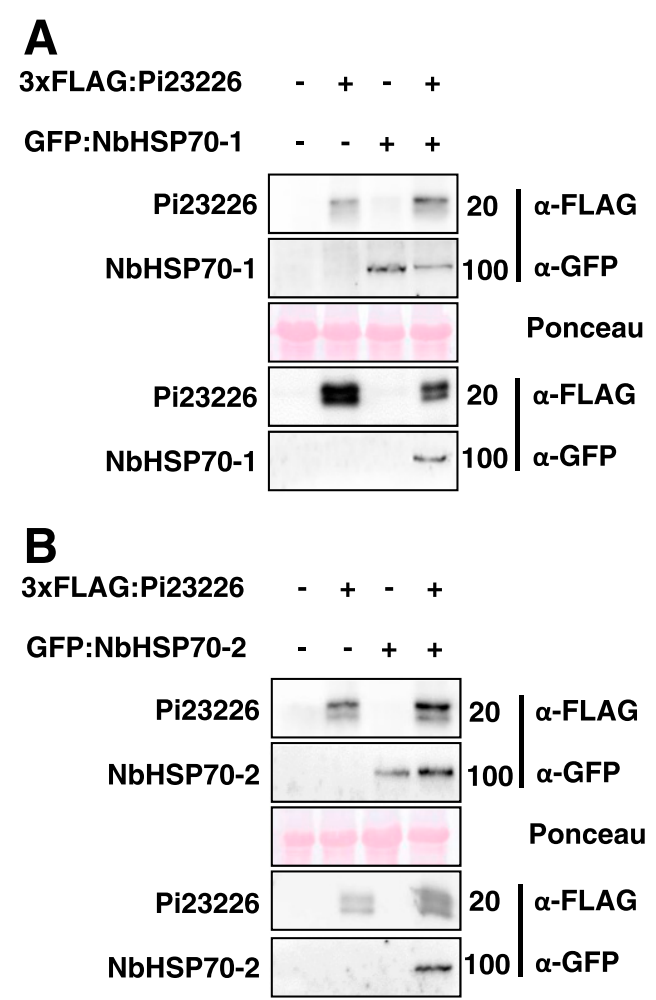

Fig. 3. NbHSP70s family members physically interact with Pi23226 in planta. $\alpha$-FLAG coimmunoprecipitation of 3xFLAG:Pi23226 with A, NbHSP70s-1 and B, NbHSP70s-2. Total protein extracts from Nicotiana benthamiana were IPed with $\alpha$-FLAG affinity gel, and precipitated proteins were immunoblotted with $\alpha$-FLAG and $\alpha$-green fluorescent protein (GFP) antibody. Untreated $N$. benthamiana leaves and single agroinfiltrated Pi23226 or NbHSP70s-1 or -2 were used as negative controls. Total protein extract of each treatment was loaded as an input and immunodetected with $\alpha$-FLAG and $\alpha$-GFP antibodies to detect the relevant protein level of each construct. All experiments were performed in three biological replicates and representative results are shown. 
cell death occurs specifically, we selected another HSP70s (Niben101Scf10036g00003.1, hereafter NbHSP70s_NC), which is distant from chosen HSP70s members, and performed coagroinfiltration with Pi23226. However, only NbHSP70s-1, not NbHSP70s_NC, hastened the cell death induced by Pi23226 (Supplementary Fig. S3).

We also performed VIGS analysis to confirm the impact of HSP70s members on the Pi23226-triggered cell death. However, in NbHSP70s-2-silenced plants, chlorophyll bleaching and a dwarf phenotype were observed (Supplementary Fig. S4).
Thus, phenotype analyses were performed in HSP70s-1silenced plants. When gene expression of NbHSP70s-1 was suppressed, Pi23226-induced cell death was compromised (Fig. 4B). These results suggest that NbHSP70s family members are involved in Pi23226-induced cell death.

To understand the role of HSP70s members on the invasion of pathogens, $P$. infestans was inoculated onto $N$. benthamiana leaves overexpressing $N b H S P 70 s-1$ or $N b H S P 70 s-2$. The empty vector used for $N b H S P 70 s-1 /-2$ constructs was infiltrated for a negative control. As a result, transient overexpression of HSP70s
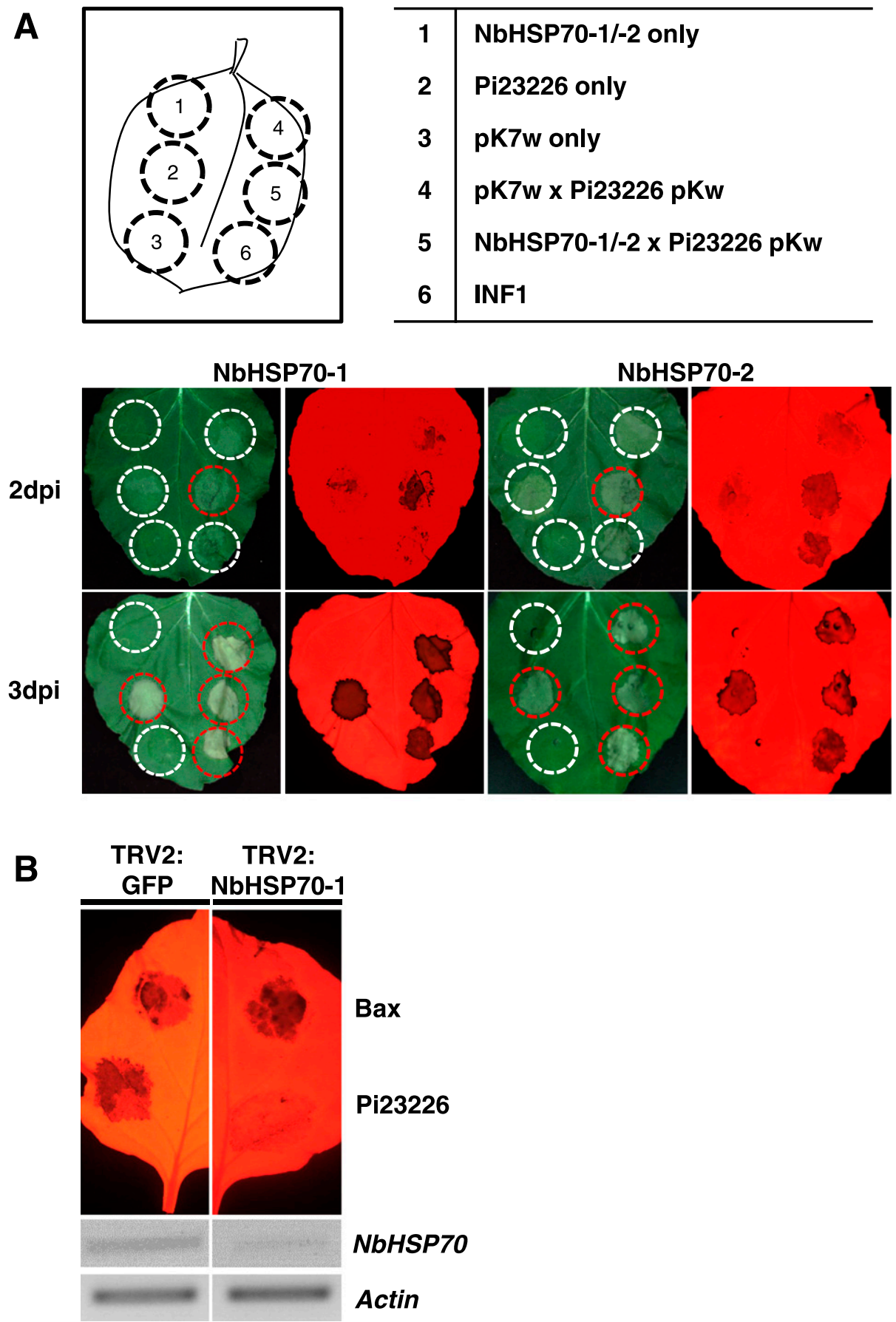

Fig. 4. NbHSP70s members accelerate Pi23226-induced cell death. A, Images show cell death in Nicotiana benthamiana of transient expression of Pi23226 with NbHSP70s members. Empty vector used for NbHSP70s expression (pK7w) and INF1 were used as a negative and positive control, respectively. Photographs were taken at 2 and 3 days postinfection (dpi). B, Images show phenotype of cell death in NbHSP70s-silenced $N$. benthamiana. Green fluorescent protein (GFP)-silenced plants were used as a negative control for virus-induced gene silencing. Bax was agroinfiltrated as a positive control for cell death response. Suppression of $\mathrm{NbHSP7Os}$ was confirmed using reverse-transcription polymerase chain reaction in comparison with gene expression of actin. All experiments were performed in more than three biological replicates and representative results are shown. 
compromised the disease symptoms of $P$. infestans on $N$. benthamiana (Fig. 5), indicating NbHSP70s members function positively in defense against $P$. infestans.

\section{Signal components of an MAPK cascade are involved in Pi23226-induced cell death.}

Previous studies have suggested that plant MAPK cascades are involved in cell death signaling mediated by pathogen infection. Furthermore, cochaperones such as HSP90 and HSP70s may function upstream of the MAPK cascade or independently in the signaling (Asai et al. 2008; Kanzaki et al. 2003; Meng and Zhang 2013). We investigated whether Pi23226-induced cell death occurs through the MAPK cascade. As general components of an MAPK cascade, an N. benthamiana MAPK-kinase, MEK2, and two MAPK, SIPK and WIPK, were selected. In VIGS analyses, Pi23226-induced cell death was compromised in $M E K 2$ - and SIPK-suppressed plants but not in $W I P K$-suppressed plants (data not shown), indicating that specific MAPK signaling components are involved in Pi23226triggered cell death (Fig. 6). Because HSP90 is known to function with HSP70s and to regulate the activity and stability of plant resistance proteins, cell death analysis was accomplished in HSP90-silenced plants by agroinfiltration of Pi23226. Consistently with previous studies, HSP90-silenced plants presented a stunted growth phenotype and compromised cell death induced by Bax as well as Pi23226 (Fig. 6). However, the cell death phenotype had no significant changes in plants
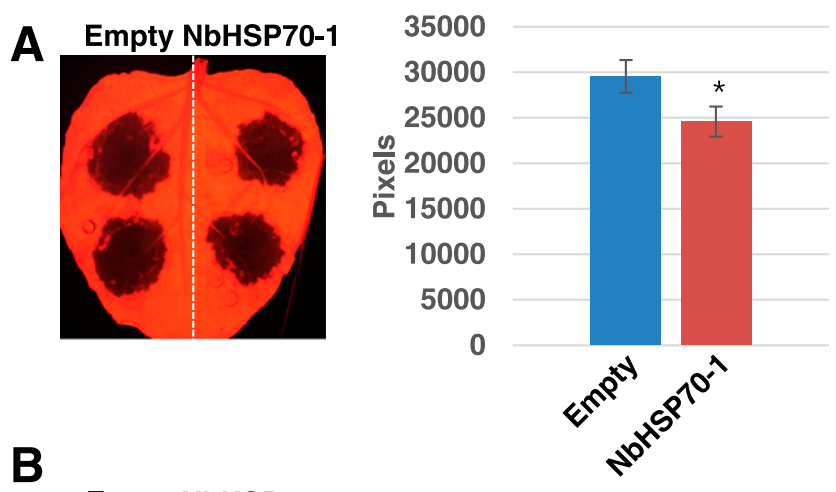

B
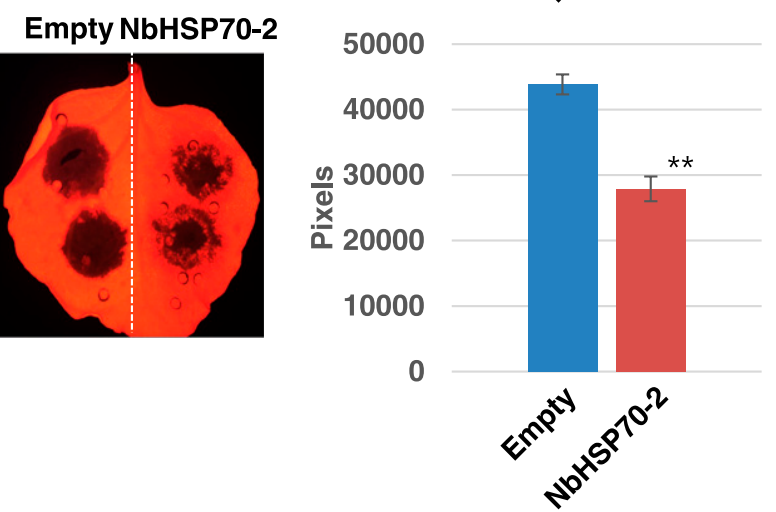

Fig. 5. NbHSP70s members suppresses pathogen growth of Phytophthora infestans. Panels on the left show representative images of the growth of $P$. infestans on Nicotiana benthamiana leaves. Empty vector used for NbHPS70 constructs was transiently expressed at on the left half of leaves as a negative control. A, NbHSP70s-1 and B, NbHSP70s-2 were agroinfiltrated on the right half of leaves. After $24 \mathrm{~h}, P$. infestans zoospores were inoculated as droplets on infiltrated leaves. Photographs were taken after approximately 4 to 6 days under the UV light. In the righthand panels, graphs represent average lesion sizes of each inoculated spot measured by ImageJ. Error bars show standard error calculated with more than 10 biological replicates. The relevance of difference was evaluated by $P$ value; $*$ indicates $P$ value $<0.05$ and $* *$ indicates $P$ value $<0.01$. suppressed for cochaperone Rarl, which is known to regulate the stability of resistance proteins together with HSP90 (Fig. 6). These results indicate that general components of an MAPK cascade and HSP90 are involved in Pi23226-induced cell death signaling.

\section{DISCUSSION}

Here, we showed that Pi23226 triggers cell death in N. benthamiana, and HSP70s members, as interactors of Pi23226, enhance the cell death. Furthermore, signaling components of an MAPK cascade such as MEK2 and SIPK are involved in the cell death. These results make us presume that the cell death induced by Pi23226 occurred by interacting with HSP70s members as the result of plant defense. As a defense factor, HSP70s may recognize Pi23226 and initiate cell death through the activation of the MAPK pathway to disturb the spread of pathogen infection. This speculation corresponds to the result in which disease symptoms of $P$. infestans were suppressed in HSP70s-overexpressed N. benthamiana.

We identified HSP70s members as interaction partners of Pi23226 using co-IP and LC-MS/MS and found that they interact with Pi23226 in planta. However, in the yeast-two-hybrid system, we could not observe the direct interaction between Pi23226 and HSP70s members (Supplementary Fig. S5). These results suggest two possibilities. First, Pi23226 might be assisted by additional components to associate with HSP70s. Noël and colleagues (2007) have found that a cochaperone, SGT1, interacts with HSP70s in vivo, whereas they did not observe the interaction between SGT1 and HSP70s using the yeast-two-hybrid system. Second, the interaction between Pi23226 and HSP70s might occur indirectly. Because HSP70s is known to form a cochaperone complex dynamically with several proteins such as HSP90 and HSP40 (Iki et al. 2012), the yeast-two-hybrid system can have limitations in representing a multiprotein complex. Although HSP70s members have been known to interact with effectors in Arabidopsis and pepper (Jelenska et al. 2010; Kim and Hwang 2015), several studies pointed out the possibility of HSP70s members as redundant and nonspecific interactors (Mellacheruvu et al. 2013; Petre et al. 2015, 2016). However, when we calculated protein scores based on the method presented by Petre and associates $(2015,2016)$, HSP70s members were ranked at the top-tier in the Pi23226-precipitated purification

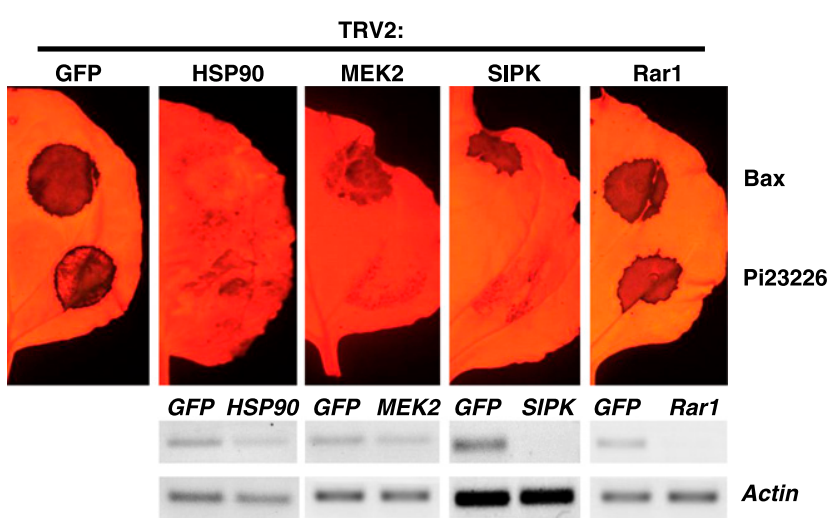

Fig. 6. General components of a mitogen-activated protein kinase cascade are involved in Pi23226-induced cell death. Virus-induced gene silencing analyses of HSP90, SIPK, and MEK2 were accomplished in Nicotiana benthamiana. Green fluorescent protein (GFP) and Rar1 were silenced for a negative and positive control, respectively. Bax was used as a positive control for cell death response. Suppression of each gene was showed using reverse-transcription polymerase chain reaction in comparison with gene expression of actin. All experiments were performed in more than three biological replicates and representative results are shown. 
results, indicating that HSP70s members could be specific interactors, at least in the case of Pi23226.

In conclusion, we found one of the most ubiquitous proteins, HSP70s, functioning in plant defense systems by regulating effector-triggered cell death. In the aspect of fitness cost, plants may utilize the most common proteins as detectors and weapons in response to the activity of pathogen effectors. However, the extent to which regulation of HSP70s interacts with an MAPK cascade in a cell death signaling pathway remains to be answered. Moreover, if HSP70s forms a complex with cochaperones, the role of the cochaperone complex in the plant immune system needs further comprehensive study.

\section{MATERIALS AND METHODS}

Plant growth conditions and pathogen inoculation.

Plants were sown and grown at approximately 22 to $25^{\circ} \mathrm{C}$ and 50 to $60 \%$ humidity, with light and dark periods of 16 and $8 \mathrm{~h}$, respectively, in a controlled walk-in chamber. After 2 weeks, seedlings were transplanted into separate plastic pots. For the pathogen virulence assay, Pi23226 pC2300 was transiently expressed on one-half of an $N$. benthamiana leaf and enhanced GFP (eGFP) pC2300 on the other. The leaves were detached at 1 day after infiltration and $P$. infestans (43072 isolate) zoospores $\left(1.0 \times 10^{5}\right.$ zoospores per milliliter) were inoculated on detached $N$. benthamiana leaves. The leaves were incubated at room temperature. After approximately 4 to 6 days, photographs were taken under white and UV light.

\section{Plasmid construction.}

The C-terminal effector domain of Pi23226 was cloned into a virus vector (pICH31160) and a binary vector (pCAMBIA2300LIC) using a ligation-independent cloning (LIC) system (Aslanidis and de Jong 1990). Then, they were transformed into a GV3101 strain for agroinfiltration.

NbHSP70s-1 and NbHSP70s-2 coding sequences were amplified from $N$. benthamiana cDNA with flanking attB sites for the GATEWAY cloning system. The polymerase chain reaction (PCR) products were introduced into pDONR221 (Invitrogen) and entry clones were recombined with a binary vector (pk7WGF2) for N-terminal eGFP fusion.

\section{Coimmunoprecipitation and immunoblot analysis.}

For coimmunoprecipitation, the protocol described by Saunders et al. (2012) was modified. In brief, total proteins were extracted from $N$. benthamiana leaves 2 days after agroinfiltration using extraction buffer (GTEN buffer $=10 \%$ [vol/vol) glycerol, $25 \mathrm{mM}$ Tris [pH 7.5], $1 \mathrm{mM}$ EDTA, $150 \mathrm{mM} \mathrm{NaCl}$ with $10 \mathrm{mM}$ dithiothreitol, $2 \%$ [wt/vol] polyvinylpolypyrrolidone, and $1 \times$ protease inhibitor cocktail). Total protein extract $(10 \mathrm{mg})$ was incubated with $\alpha$-FLAG affinity gel (BioLegend) for $2 \mathrm{~h}$ at $4^{\circ} \mathrm{C}$. After washing three times with cold GTEN buffer including 0.1\% Tween-20, the precipitated proteins were eluted by boiling with Laemmli sample buffer (Laemmli 1970). Immunoblot analyses were performed on sodium dodecyl sulfate polyacrylamide gel electrophoresis. Monoclonal ANTI-FLAG M2 antibody (SigmaAldrich) and anti-GFP antibody (Abcam) were used at 1:20,000 dilution, and antimouse antibody (Thermo Fisher Scientific) were used at 1:25,000 as a secondary antibody. Protein bands were detected using ECL reagent (GE healthcare).

\section{Agroinfiltration assay.}

For in planta transient expression, an Agrobacterium sp. carrying an appropriate construct was inoculated overnight in yeast extract peptone medium containing selective antibiotics at $28^{\circ} \mathrm{C}$. Incubated culture was centrifuged at 3,000 rpm for $8 \mathrm{~min}$ and resuspended in infiltration buffer $\left(10 \mathrm{mM}\right.$ MES, $10 \mathrm{mM} \mathrm{MgCl}_{2}$, and $150 \mu \mathrm{M}$ acetosyringone) and diluted to a final concentration at an optical density at $600 \mathrm{~nm}$ of 0.5 . For efficient transformation, the suspension was incubated at room temperature for $2 \mathrm{~h}$ and infiltrated into approximately 4- to 5-week-old $N$. benthamiana using a needless syringe.

\section{Tobacco rattle virus (TRV)-based VIGS in N. benthamiana.}

VIGS constructs were made by cloned PCR fragments from HSP70s- 1 and HSP70s-2 using provided primer sets into TRV vectors (Supplementary Table S1). Agrobacterium containing the VIGS construct was infiltrated into approximately 3- to 4week-old $N$. benthamiana. TRV constructs expressing GFP and phytoene desaturase were used as controls for gene expression and successful VIGS, respectively. After 2 weeks, suppression of corresponding gene expression was confirmed by reversetranscription (RT)-PCR and further experiments were performed.

\section{RT-PCR.}

RNA was extracted from $N$. benthamiana leaves using TRIZOL reagents (Thermo Fisher Scientific) and cDNA was synthesized from the extracted RNA using SuperScript II RT (Thermo Fisher Scientific). RT-PCR was performed using specific primer sets which amplify partial regions of genes.

\section{ACKNOWLEDGMENTS}

We thank S. Kamoun in the Sainsbury Laboratory, United Kingdom for a kind gift of the $P$. infestans effector construct.

\section{LITERATURE CITED}

Asai, S., Ohta, K., and Yoshioka, H. 2008. MAPK signaling regulates nitric oxide and NADPH oxidase-dependent oxidative bursts in Nicotiana benthamiana. Plant Cell 20:1390-1406.

Aslanidis, C., and de Jong, P. J. 1990. Ligation-independent cloning of PCR products (LIC-PCR). Nucleic Acids Res. 18:6069-6074.

Bozkurt, T. O., Schornack, S., Win, J., Shindo, T., Ilyas, M., Oliva, R., Cano, L. M., Jones, A. M., Huitema, E., van der Hoorn, R. A., and Kamoun, S. 2011. Phytophthora infestans effector AVRblb2 prevents secretion of a plant immune protease at the haustorial interface. Proc. Natl. Acad. Sci. U.S.A. 108:20832-20837.

Bozkurt, T. O., Richardson, A., Dagdas, Y. F., Mongrand, S., Kamoun, S., and Raffaele, S. 2014. The plant membrane-associated REM1. 3 remorin accumulates in discrete perihaustorial domains and enhances susceptibility to Phytophthora infestans. Plant Physiol. 165:1005-1018.

Cooke, D. E. L., Cano, L. M., Raffaele, S., Bain, R. A., Cooke, L. R., Etherington, G. J., Deahl, K. L., Farrer, R. A., Gilroy, E. M., Goss, E. M., Grünwald, N. J., Hein, I., MacLean, D., McNicol, J. W., Randall, E., Oliva, R. F., Pel, M. A., Shaw, D. S., Squires, J. N., Taylor, M. C., Vleeshouwers, V. G. A. A., Birch, P. R. J., Lees, A. K., and Kamoun, S. 2012. Genome analyses of an aggressive and invasive lineage of the Irish potato famine pathogen. PLoS Pathog. 8:e1002940.

Haas, B. J., Kamoun, S., Zody, M. C., Jiang, R. H., Handsaker, R. E., Cano, L. M., and Bozkurt, T. O. 2009. Genome sequence and analysis of the Irish potato famine pathogen Phytophthora infestans. Nature 7262:393-398.

Haverkort, A. J., Boonekamp, P. M., Hutten, R., Jacobsen, E., Lotz, L. A. P., Kessel, G. J. T., and Van der Vossen, E. A. G. 2008. Societal costs of late blight in potato and prospects of durable resistance through cisgenic modification. Potato Res. 1:47-57.

Iki, T., Yoshikawa, M., Meshi, T., and Ishikawa, M. 2012. Cyclophilin 40 facilitates HSP90-mediated RISC assembly in plants. EMBO J. 2:267-278

Jelenska, J., van Hal, J. A., and Greenberg, J. T. 2010. Pseudomonas syringae hijacks plant stress chaperone machinery for virulence. Proc. Natl. Acad. Sci. U.S.A. 107:13177-13182.

Jones, J. D. G., and Dangl, J. L. 2006. The plant immune system. Nature 444:323-329.

Kanzaki, H., Saitoh, H., Ito, A., Fujisawa, S., Kamoun, S., Katou, S., Yoshioka, H., and Terauchi, R. 2003. Cytosolic HSP90 and HSP70 are essential components of INF1-mediated hypersensitive response and non-host resistance to Pseudomonas cichorii in Nicotiana benthamiana. Mol. Plant Pathol. 4: 383-391.

Kim, N. H., and Hwang, B. K. 2015. Pepper heat shock protein 70a interacts with the type III effector AvrBsT and triggers plant cell death and immunity. Plant Physiol. 167:307-322. 
Kim, S. B., Lee, H. Y., Seo, S., Lee, J. H., and Choi, D. 2015. RNAdependent RNA polymerase (NIb) of the potyviruses is an avirulence factor for the broad-spectrum resistance gene Pvr4 in Capsicum annиum cv. CM334. PloS One 3:e0119639.

King, S. R. F., McLellan, H., Boevink, P. C., Armstrong, M. R., Bukharova, T., Sukarta, O., Win, J., Kamoun, S., Birch, P. R. J., and Banfield, M. J. 2014. Phytophthora infestans RXLR effector PexRD2 interacts with host MAPKKK $\varepsilon$ to suppress plant immune signaling. Plant Cell 26:1345-1359.

Laemmli, U. K. 1970. Cleavage of structural proteins during the assembly of the head of bacteriophage T4. Nature 227:680-685.

McLellan, H., Boevink, P. C., Armstrong, M. R., Pritchard, L., Gomez, S., Morales, J., Whisson, S. C., Beynon, J. L., and Birch, P. R. J. 2013. An RxLR effector from Phytophthora infestans prevents re-localisation of two plant NAC transcription factors from the endoplasmic reticulum to the nucleus. PLoS Pathog. 9:e1003670.

Mellacheruvu, D., Wright, Z., Couzens, A. L., Lambert, J. P., St-Denis, N. A., Li, T., Miteva, Y. V., Hauri, S., Sardiu, M. E., Low, T. Y., Halim, V. A., Bagshaw, R. D., Hubner, N. C., Al-Hakim, A., Bouchard, A., Faubert, D., Fermin, D., Dunham, W. H., Goudreault, M., Lin, Z.-Y., Badillo, B. G., Pawson, T., Durocher, D., Coulombe, B., Aebersold, R., Superti-Furga, G., Colinge, J., Heck, A. J. R., Choi, H., Gstaiger, M., Mohammed, S., Cristea, I. M., Bennett, K. L., Washburn, M. P., Raught, B., Ewing, R. M., Gingras, A.-C., and Nesvizhskii, A. I. 2013. The CRAPome: A contaminant repository for affinity purification-mass spectrometry data. Nat. Methods 10: 730-736.

Meng, X., and Zhang, S. 2013. MAPK cascades in plant disease resistance signaling. Annu. Rev. Phytopathol. 51:245-266.

Nausch, H., Mikschofsky, H., Koslowski, R., Meyer, U., Broer, I., and Huckauf, J. 2012. High-level transient expression of ER-targeted human interleukin 6 in Nicotiana benthamiana. PLoS One 11:e48938.
Noël, L. D., Cagna, G., Stuttmann, J., Wirthmüller, L., Betsuyaku, S., Witte, C.-P., Bhat, R., Pochon, N., Colby, T., and Parker, J. E. 2007. Interaction between SGT1 and cytosolic/nuclear HSC70 chaperones regulates Arabidopsis immune responses. Plant Cell 19:4061-4076.

Petre, B., Saunders, D. G. O., Sklenar, J., Lorrain, C., Krasileva, K. V., Win, J., Duplessis, S., and Kamoun, S. 2016. Heterologous expression screens in Nicotiana benthamiana identify a candidate effector of the wheat yellow rust pathogen that associates with processing bodies. PLoS One 11:e0149035

Petre, B., Saunders, D. G. O., Sklenar, J., Lorrain, C., Win, J., Duplessis, S., and Kamoun, S. 2015. Candidate effector proteins of the rust pathogen Melampsora larici-populina target diverse plant cell compartments Mol. Plant-Microbe Interact 28:689-700.

Saunders, D. G. O., Breen, S., Win, J., Schornack, S., Hein, I., Bozkurt, T. O., Champouret, N., Vleeshouwers, V. G., Birch, P. R. J., Gilroy, E. M., and Kamoun, S. 2012. Host protein BSL1 associates with Phytophthora infestans RXLR effector AVR2 and the Solanum demissum Immune receptor $\mathrm{R} 2$ to mediate disease resistance. Plant Cell 24: 3420-3434.

Zheng, X., McLellan, H., Fraiture, M., Liu, X., Boevink, P. C., Gilroy, E. M., Chen, Y., Kandel, K., Sessa, G., Birch, P. R. J., and Brunner, F. 2014. Functionally redundant RXLR effectors from Phytophthora infestans act at different steps to suppress early flg22-triggered immunity. PLoS Pathog. 10:e1004057.

\section{AUTHOR-RECOMMENDED INTERNET RESOURCES}

Nicotiana benthamiana draft genome sequence: https://solgenomics.net/organism/Nicotiana_benthamiana/genome 